\title{
Retention of ${ }^{90} \mathrm{Y}$ in patients with rheumatoid arthritis
}

\author{
N. W. RAMSEY \\ Department of Medical Physics, Charing Cross Hospital, London
}

The use of radioactive compounds for the treatment of patients with chronic rheumatoid effusion has been proceeding in the Charing Cross Hospital Group since 1968 . Initially, a colloidal suspension of radioactive gold $\left({ }^{198} \mathrm{Au}\right)$ stabilized with gelatin and glucose was used, and the results in a group of patients whose knees were treated in this way have been published (Grahame, Ramsey, and Scott, 1970).

Subsequently, substances incorporating ${ }^{90} \mathrm{Y}$ in place of ${ }^{198} \mathrm{Au}$ were used because of the greater penetration of the $\beta$-radiation and the absence of a $\gamma$ ray component with its incidental irradiation of the gonads. This paper gives the results of observations that have been made of the retention of ${ }^{90} \mathrm{Y}$ compounds used for treating knee effusions. Nine patients were treated, two with a resin colloid preparation, and seven with colloidal yttrium silicate.

The patients treated with the resin colloid suspension complained of pain in the knee joint. Furthermore, it was discovered that the resin colloid preparation was, to some extent, unstable, and the use of this compound was discontinued. Thereafter, treatments were given with colloidal yttrium silicate, and none of the patients complained of discomfort as a result of the injection.

\section{Material and methods}

The knee joint spaces were flushed out beforehand with normal saline to remove fibrin debris, an interval of 10 days being allowed for healing before the injection.

Both preparations of ${ }^{90} \mathrm{Y}$ were obtained from the Radiochemical Centre, Amersham. In all cases, the dose given was $5 \mathrm{mCi}$., in a total volume of $10 \mathrm{ml}$., the original preparation being diluted with normal saline. The needle used for injection and the needle track in the tissues were flushed with saline as the needle was withdrawn after instillation.

The knee being treated was immobilized with a moulded splint, placed posteriorly on the leg, for 1 week after the injection.

Measurements were made by two methods:

(1) A Mecaserto single-headed scanner was used in an attempt to locate activity. Good results were obtained over the knee, but no response could be obtained over the trunk, because of the extremely low intensity of the Bremsstrahlen emitted. Later, an Elscint double-headed scanner was used, and the response obtained was very similar.

(2) The patient's knee was placed between a pair of identical scintillation counters fitted with collimators, the acceptance angles of which were large enough to ensure that radiation from any part of the knee joint would be received.

The activity assessed using a scanner was taken to be proportional to the total number of pulses recorded over the knee, with appropriate allowances for instrumental factors, such as line spacing and speed of travel.

The activity measured with the pair of counters was recorded by means of a scaler, 10,000 counts being registered, so that the standard deviation of the count was 1 per cent. The relative positions of the collimators were maintained constant throughout, the separation between the crystal faces being $42 \mathrm{~cm}$., which was large compared with the knee diameters. With a pair of identical detectors used in this way, the activity measured is taken as being proportional to ${ } C_{1} C_{2}$, where $C_{1}$ and $C_{2}$ are the count rates recorded in the two detectors. This formula considerably reduces the dependence of the recorded count rate on the position of the ${ }^{90} \mathrm{Y}$ in the knee joint as compared with the result obtained by adding the two count rates.

Measurements were made at 1 and 7 hours after instillation, and thereafter at daily intervals for 1 week. Immediately before each measurement, the equipment was standardized by recording the activity of a given quantity of ${ }^{90} \mathrm{Y}$, dispensed at the same time as the dose was prepared. A filter of $3 \mathrm{~cm}$. of tissue-equivalent wax was inserted between this source and the detectors during its measurement, so that the intensity of Bremsstrahlen produced was reproducible. By comparing the count rate from the knee with that from the standardizing source, no correction was necessary for radioactive decay.

\section{Results}

The results obtained for any one patient using the pair of opposed counters were more consistent than those from either of the scanners, and where disagreement was found, results by the former method were accepted. 
Figs 1 to 5 illustrate some of the results obtained for the silicate material. Results for the resin colloid were erratic, for reasons which are not quite clear, but they indicated that the amount escaping from the knee joint was about the same as for the silicate. Fig. 6 shows a result for resin colloid retention measured by scanner. The amount of activity remaining in the knee, corrected for radioactive decay, is plotted against time in all six Figures. The observations show that, where an initial escape of activity occurs from the knee, this is complete within approximately 24 hours.

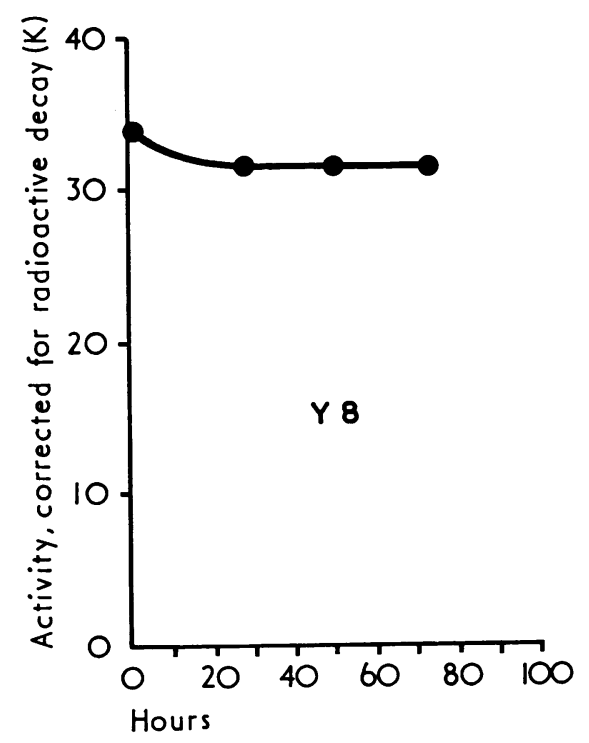

FIG. 1

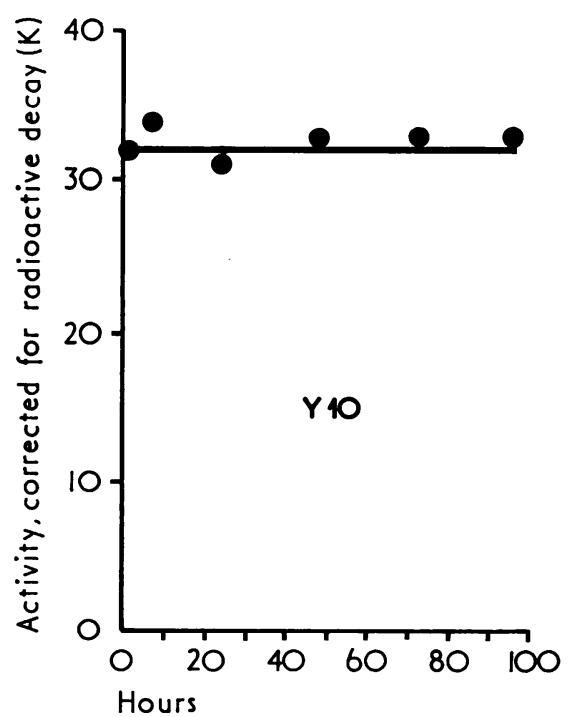

FIG. 2
The percentage retention in the knee joint ranged from 100 to 80 per cent. (mean 91 per cent.).

\section{Discussion}

It thus appears that both the ${ }^{90} \mathrm{Y}$ silicate and the colloidal resin preparations escape from the site of injection just as colloidal ${ }^{198} \mathrm{Au}$ is known to do (Ramsey and Scott, in preparation). Furthermore, the pattern of escape, i.e. approaching completion within 24 hours, is similar.

Noteworthy also is the wide variation in degree of retention, a feature already reported on by several authors for colloidal ${ }^{198} \mathrm{Au}$ (e.g. Virkkunen, Krusius,

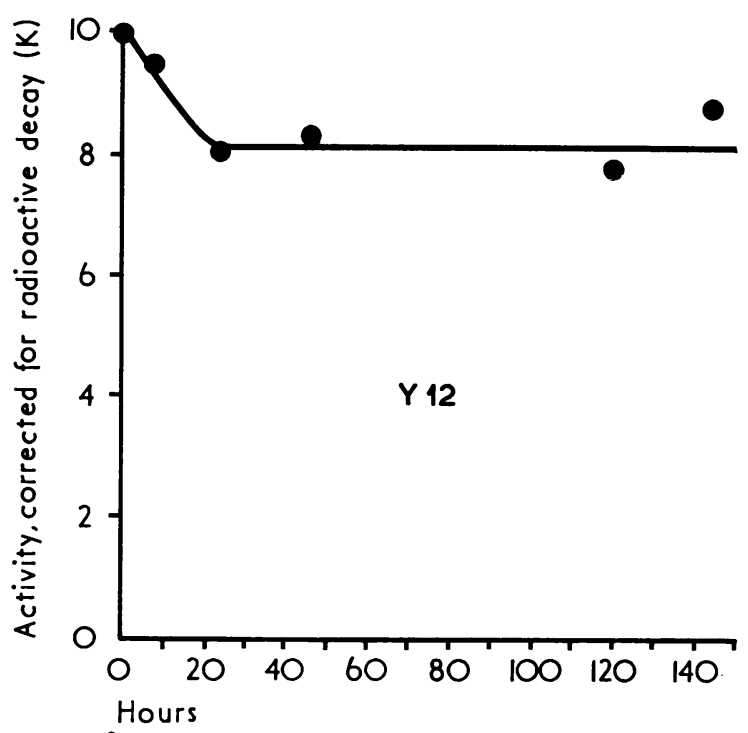

FIG. 3

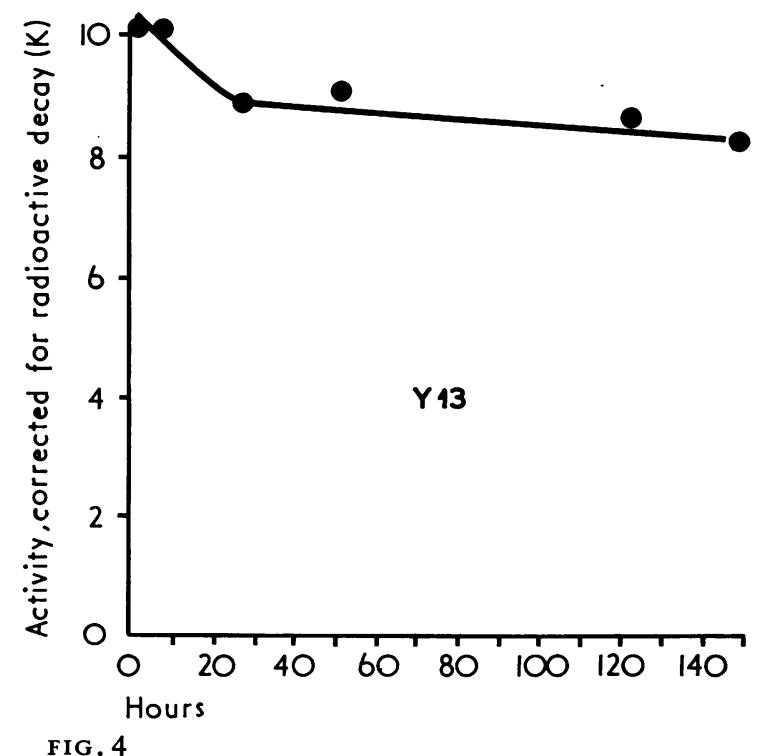




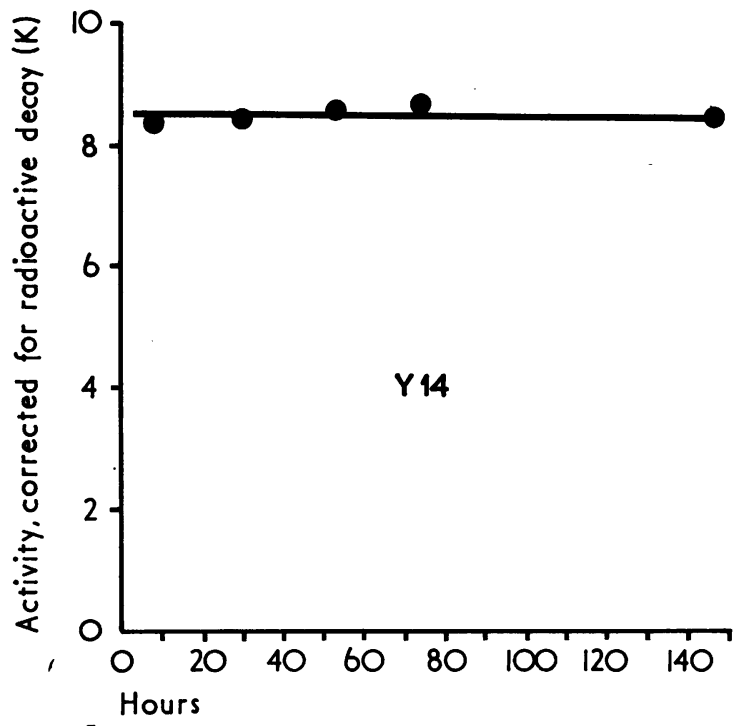

FIG. 5

FIGS 1 To 5 Activity in the knee expressed as counts per second, and corrected for radioactive decay, plotted against time after injection of colloidal ${ }^{90} \mathrm{Y}$ silicate

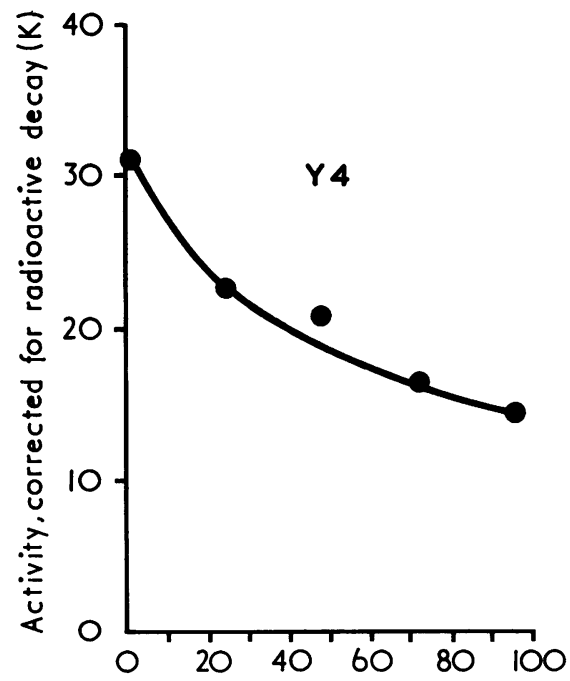

Hours

FIG. 6 Activity in the knee expressed as counts per second, and corrected for radioactive decay, plotted against time after injection of ${ }^{90} \mathrm{Y}$ resin colloid

and Heiskanen, 1967; Grahame and others, 1970). The variation is so large, ranging from 100 to about 75 per cent. for ${ }^{198} \mathrm{Au}$, and from 100 to 80 per cent. for ${ }^{90} \mathrm{Y}$, that the cause of escape would appear to depend more on factors related to the state of the knee joint, than on the material injected.

\section{Discussion}

DR. GOODE In eight outpatients I have tried lavage on knee joints on two or three occasions to see if this would be beneficial. This was done through a trocar with kneading of the synovium. They were done serially at 7 to 14 day intervals. The amount of fibrin removed decreased only slightly from the first to the second and second to the third occasion. So I think that an interval of 10 days, although it might be beneficial from some points of view, may not benefit the amount of fibrin left in the knee joint. If you are going to use this as a preliminary to the administration of radioactive colloids, what you are actually doing needs to be more accurately measured. DR. YATES The lavage performed in these patients 10 days before treatment with ${ }^{90} \mathrm{Y}$ was for arthroscopy and by using a large quantity of saline and a $5 \mathrm{~mm}$. cannula, we could see that we had cleared the joint of loose fibrin. In a certain number of control patients, who were again examined by arthroscopy, fibrin production was very small.

DR. ROBERTS Do you think that the early fall in retention of isotope represents a change in the distribution of the isotope within the joint rather than disappearance from the joint. Do you think that perhaps the yttrium may be in a slightly different situation after 24 hours, but still in the knee?

DR. RAMSEY I do not think so because we ensured that the acceptance angles of the collimators was more than adequate to cover the knee and we used the formula, the square root of the two count rates, rather than their sum. This is a very rough approximation to account for the activity anywhere inside the knee joint; in other words it takes account of the tissue absorption of radiation.

PROFESSOR INGRAND A comment about resin colloid for Dr. Peake from Amersham. When we deal with anion or cation exchange resins, do you think it possible that yttrium, which has been introduced by exchange, may be displaced by the cations of biological fluid $\left(\mathrm{Ca}^{++}, \mathrm{Na}^{++}\right.$, etc. $)$?

DR. PEAKE I think this is certainly a possibility that must be borne in mind. Yttrium would tend to be more strongly held than sodium or potassium as it is highly charged. It might also be worth mentioning that the general weight of information that has come to us at the Radiochemical Centre from users of the various colloids of yttrium leads us to believe that yttrium resin colloid tended to be more stable in knee joints and to leak less than the silicate. DR. RAMSEY I think I can only comment that, in view of the very wide variation with gold and yttrium compounds-anything between 0 and 30 per cent., it is difficult to draw any firm conclusions unless you have very large numbers of patients. 